\title{
Creation and testing of a practical visual function assessment for use in Africa: correlation with visual acuity, contrast sensitivity, and near vision in Malawian adults
}

Karin van Dijk, Susan Lewallen, Moses Chirambo, Jane Gardiner, Brian Hoar, Janette Lindley, N Kevin Wade, Paul Courtright

Christoffel

Blindenmission, Boshof 10, 7873 AC

Odoorn, Netherlands

K van Dijk

BC Centre for

Epidemiologic and

International

Ophthalmology,

University of British

Columbia, St Paul's

Hospital, Vancouver,

Canada

S Lewallen

J Gardiner

B Hoar

$\mathrm{J}$ Lindley

N K Wade

P Courtright

Sight Savers

International, PO Box

30858, Lilongwe, 3 ,

Malawi

M Chirambo

Correspondence to:

Dr Paul Courtright, BC

Centre for Epidemiologic

and International

Ophthalmology, St Paul's

Hospital, 1081 Burrard

Street, Vancouver, BC V6Z

1Y6, Canada.

Accepted for publication 27 January 1999

\begin{abstract}
Aim-To develop and test a practical visual function assessment for use in developing countries.

Methods-Using focus group discussions and interviews with eyecare workers and low vision specialists in Malawi, 13 questions related to visual characteristics of activities of daily living were designed. Patients presenting to an eye clinic were recruited and interviewed. Visual acuity, near vision, and contrast sensitivity were measured. Analysis sought to determine the degree of correlation between the vision indices and visual function.

Results-The visual function questionnaire was easy to administer. Visual function correlated with visual acuity, contrast sensitivity, near vision, and patient reported visual problem. People with a higher frequency of "not applicable" responses had lower visual function scores. Multivariate modelling revealed that visual acuity and number of questions felt to be applicable were independently associated with visual function. Reducing the questionnaire to nine questions did not affect the degree of correlation with any of the visual indices.

Conclusion-The authors' visual function assessment correlates well with different measures of visual acuity. People with reduced vision for a prolonged period may no longer consider doing certain tasks and the number of questions considered appropriate by an individual may be an additional measure of visual function. Assessment of visual function by health workers may be a valuable tool in improving surgical uptake by encouraging both health personnel and patients to recognise that they have difficulties undertaking activities of daily living as well as a measure of monitoring and evaluating cataract outcomes.
\end{abstract}

(Br F Ophthalmol 1999;83:792-795)

There is growing recognition that measurement of a person's ability to perform activities of daily living which require vision is more meaningful than measurement of his distance visual acuity. The distance visual acuity measurement has been used extensively to evaluate vision because it is relatively simple and objective. Visual acuity is widely used to define visual disability, blindness (although all definitions do not use the same level of visual acuity), surgical success rates, and levels at which cataract surgery is deemed necessary by insurers; some of these levels are arbitrarily defined and tell little about a patient's ability to function.

Recently, there has been interest in designing questionnaires to measure visual function. ${ }^{1-4}$ These have been designed primarily to monitor the outcome of cataract surgery in developed countries $^{5-7}$ and they are not appropriate for all populations. ${ }^{8}$ Visual function is a measure of activities of daily living affected by vision while quality of life indices convey a more comprehensive picture of the burden of vision $\operatorname{loss}^{9}{ }^{10}$; measurement of the latter was not the intent of this study.

Assessments of visual function would be useful in populations in developing countries for several purposes - to guide referral from the field, to assist in defining blindness and visual disability more meaningfully (perhaps on a local level), to monitor the success of campaigns to reduce blindness and visual disability at the community level, and to promote the value of surgical intervention to policy makers and patients. With these purposes in mind we developed and (pilot) tested a simple visual function assessment appropriate for use in different developing countries.

\section{Methods}

Thirteen questions were designed based on interviews with local eyecare workers and low vision specialists, focus groups ${ }^{11}$ with patients on a hospital eye ward, and review of existing visual function questionnaires. Care was taken to include questions relevant to men and women, rural and urban dwellers, and to include tasks related to near and distance acuity and contrast sensitivity. The questions are shown in Table 1 . All questions were designed to be answered on a four point scale $(0$, no problem, 1 , a little problem, 2 , a lot of problem, 3 , or cannot do at all) or deemed not applicable by the patient. If an individual reported that the question was not appropriate no score was given. Scores for all questions were totalled and divided by the total number of questions answered. This value was multiplied by 33.3 . Thus, the range in scores was 0 to 100 , a high score indicating decreased visual function. 
Table 1 Visual function questions

Do you have any difficulty reading?

Do you have any difficulty recognising faces?

Do you have any difficulty telling the difference between a K 10 and a K 20 note because of your eyesight?

Do you have any difficulty walking around at night because of your eyesight?

Do you have any difficulty recognising food on your plate?

Do you have any difficulty avoiding potholes, stones, or branches when you are walking because of your eyesight?

Do you have any difficulty cooking, chopping vegetables, or pouring water because of your eyesight?

Do you have any difficulty using the latrine without assistance because of your eye sight?

Do you have any difficulty participating in social activities like weddings or funerals because of your eyesight?

Do you have any difficulty sorting stones from rice or beans because of your eyesight?

Do you have any difficulty sewing or making baskets or mats because of your eyesight?

Do you have any difficulty tending your garden because of your eyesight?

Do you have any difficulty repairing your house because of your eyesight?

Potential study subjects were recruited from among patients presenting to the Queen Elizabeth Central Hospital Eye Clinic, Blantyre, Malawi, one morning per week over a 16 week period. Only patients $\geqslant 45$ years of age were enrolled. On enrolment, patients were asked the reason for presentation; information on residency, occupation, age, and sex was also collected. This was followed by a question regarding their perception of their vision. All questionnaires were administered by one person. The patient's distance and near visual acuity were measured using $\log M A R$ with illiterate $\mathrm{E}$ charts with the spectacle correction a study subject normally used. Patients were allowed to hold the near card at the distance they were comfortable and the smallest line on which they could correctly identify the direction of the Es was recorded, as well as the distance at which they held the card. Both distance and near vision were measured in the same room with the same illumination in all patients. Contrast sensitivity was also measured under identical conditions for all patients, using the Lea-Screener (Precision Vision, Ltd) at 1 metre. This test uses four symbols which are recognised in Malawi, verified by asking patients to name the symbols before testing. Contrast sensitivity was not measured in eyes with distance visual acuity less than $1 / 60$ as we found this was not productive.

Clinical examination was performed using a torch and direct ophthalmoscope (undilated examination). The following were assessed and recorded on a standard form-pupil reaction, corneal clarity, quality of red reflex, examination of optic nerve, and macula. The examining ophthalmologist was asked to assess whether the clinical examination was consistent with the visual acuity and to determine the cause of decreased vision in any eye with distance acuity of $6 / 18$ or less. Four different ophthalmologists were responsible for the clinical examinations with overlap between each successive examiner to decrease interobserver variations.

In 13 patients a second (identical) visual function interview was conducted 2-4 weeks after the first for determining interobserver variability.

Data were included in an SPSS-PC+ data file. Univariate analysis included Student's $t$ tests (and ANOVA) for continuous variables and $\chi^{2}$ statistics for dichotomous variables. Pearson's correlation coefficients were used for determining the degree of correlation between these vision indices and visual function. A linear regression model was created to determine which vision related indices were independently associated with visual function. The study was approved the Malawi health sciences research committee.

\section{Results}

A total of 163 patients who fit the criteria were enrolled in the study. Two thirds of the enrollees were male, reflecting the typical underrepresentation of females presenting for treatment. The mean age was 60.4 years (SD 11.9) for males and 60.1 years (12.5) for females. Six patients $(3.7 \%)$ were blind (visual acuity $<3 / 60$ in the better eye), $53(20.8 \%)$ were visually impaired (visual acuity 3/60-6/18), and 104 $(63.8 \%)$ had a vision better than 6/18. The mean visual acuity (logMAR) was -0.355 (range 0 to -2.3 ). Overall, 80 patients presented to the clinic with vision related problems, 76 with an eye problem (not vision related), and seven with no reported eye complaint.

Using all 13 questions of the visual function assessment, the mean VF score was 35.1, ranging from 0 to 100 . Visual function correlated with visual acuity, contrast sensitivity, near vision, and patient self reported visual problem

Table 2 Visual function according to visual indices

\begin{tabular}{|c|c|c|c|}
\hline & \multicolumn{3}{|c|}{ Visual function score } \\
\hline & No & Mean $(S D)$ & $p$ Value \\
\hline \multicolumn{4}{|l|}{ Visual acuity } \\
\hline$>=6 / 18$ & 104 & $20.6(16.9)$ & \\
\hline $6 / 24-3 / 60$ & 53 & $36.2(21.7)$ & \\
\hline$<3 / 60$ & 6 & $55.8(22.2)$ & $<0.001$ \\
\hline \multicolumn{4}{|l|}{ Contrast sensitivity } \\
\hline 0 & 30 & $48.1(27.3)$ & \\
\hline 1 & 15 & $32.4(16.8)$ & \\
\hline 2 & 20 & $34.8(12.4)$ & \\
\hline 3 & 24 & $21.3(12.3)$ & \\
\hline 4 & 32 & $21.0(15.4)$ & \\
\hline 5 & 42 & $14.1(13.0)$ & $<0.001$ \\
\hline \multicolumn{4}{|l|}{ Near vision } \\
\hline 8 & 47 & $15.7(12.8)$ & \\
\hline 20 & 94 & $26.9(18.5)$ & \\
\hline 48 & 14 & $44.4(22.1)$ & $<0.001$ \\
\hline \multicolumn{4}{|l|}{ Vision OK } \\
\hline Yes & 134 & $24.5(19.5)$ & \\
\hline No & 28 & $36.8(21.3)$ & 0.008 \\
\hline \multicolumn{4}{|l|}{ Questions answered } \\
\hline$<=9$ & 7 & $42.3(26.3)$ & \\
\hline 10 & 13 & $32.8(15.1)$ & \\
\hline 11 & 42 & $32.2(23.9)$ & \\
\hline 12 & 50 & $24.5(18.9)$ & \\
\hline 13 & 51 & $21.6(18.6)$ & 0.02 \\
\hline \multicolumn{4}{|l|}{ Reason for presentation } \\
\hline Vision related complaint & 80 & $32.5(20.3)$ & \\
\hline \multicolumn{4}{|l|}{ Non-vision related } \\
\hline complaint & 76 & $22.5(20.5)$ & \\
\hline No eye complaint & 7 & $12.6(10.1)$ & $<0.001$ \\
\hline
\end{tabular}


Table 3 Correlation with visual function: findings from logistic regression analysis

\begin{tabular}{lcr}
\hline Variable & $\begin{array}{l}\text { Standardised } \\
\text { coefficient }\end{array}$ & \multicolumn{1}{c}{$t$ (p value) } \\
\hline Age & 0.03 & $0.33(0.70)$ \\
Visual acuity (logMAR) & -0.27 & $-2.47(0.02)$ \\
Contrast sensitivity & -0.17 & $-1.36(0.18)$ \\
Items answered & -0.17 & $-2.43(0.02)$ \\
Near vision & 0.14 & $1.70(0.09)$ \\
\hline
\end{tabular}

Table 4 Correlation between visual acuity findings and visual function

\begin{tabular}{llc}
\hline & \multicolumn{2}{l}{ Visual function score } \\
\cline { 2 - 3 } & All 13 questions & 9 questions \\
\hline Visual acuity (logMAR) & -0.52 & -0.51 \\
Contrast sensitivity & -0.56 & -0.57 \\
Near vision & 0.41 & 0.42 \\
Questions answered & -0.26 & -0.24 \\
\hline \multirow{2}{*}{ Pearson's correlation $\mathrm{p}$ value $<0.001$ for all variables. }
\end{tabular}

(Table 2). We also found that patients who reported more questions as "not applicable" had a lower visual function score.

The linear regression model (with visual function as the outcome) revealed that both visual acuity $(\log M A R)$ and number of questions felt to be applicable were independently associated with visual function (Table 3 ). The domains with the highest proportion of "not applicable" were repairing the house $(40.5 \%)$, cooking $(36.8 \%)$, and reading $(20.2 \%)$. Women were more likely to not respond to the questions related to house repairs and reading, while men were more likely to not respond to the cooking questions. There was no difference between males and females in the overall visual function score or the number of questions answered.

Because we felt that 13 questions may be too lengthy for routine use in a population based setting, four questions were selected for exclusion. These included the questions on latrine use, recognising food, and gardening (because of the few problems reported), and repairing the house (because of the high number reporting not applicable). The cooking and reading questions were kept in the assessment because of the high value placed on cooking by adult women and the status associated with reading by the society. The recalculated visual function score was compared with the original (13 question) visual function score. There was a high degree of agreement $(r=0.98)$ and similar correlation with the measures of visual acuity, contrast sensitivity, and near vision (Table 4 ).

There was a high degree of internal consistency in the 13 people who had two independent visual function assessments $(r=0.86$, alpha $=0.92$ ). Also, there was good consistency between questions specifically related to near distance tasks $\left(\chi^{2}=44.9, \mathrm{p}<0.001\right.$ comparing near vision with reading, and $\chi^{2}=26.2$, $\mathrm{p}<0.001$ for sorting) and questions related to contrast sensitivity (ANOVA $\mathrm{F}=17.0$, $\mathrm{p}<0.001$ comparing contrast sensitivity and recognising faces and ANOVA $F=7.0$, $\mathrm{p}<0.001$ for avoiding potholes)

\section{Discussion}

Because there is no "gold standard" for validating a visual function assessment, other researchers have relied on comparing visual function scores with the distance visual acuity and quality of life measures. Although we have no quality of life measures for this population, it is reassuring to find that our visual function scores are correlated with distance visual acuity, near vision, and contrast sensitivity. Also, adequate internal consistency was demonstrated through matched assessments.

It is interesting that a relatively simple contrast sensitivity measure correlated well with the visual function score, suggesting that contrast sensitivity, which plays an important role in recognising faces or detecting potholes, is as important a measure of vision as distance visual acuity.

Although the use of different questionnaires in different countries may make comparisons between countries more difficult, our experience indicates that it is important to make questions locally appropriate. We suggest designing questions which fit into three categories-personal care, mobility and social interaction, and income (or self sufficiency) generation. These should include near and distance tasks and take into account the need for contrast sensitivity. As much as possible, questions should be designed to be applicable to both males and females, however, sex specific questions of significant value in the culture should not be neglected. Questions applicable to both rural and urban dwellers should also be considered.

Our finding of an association between a large number of "not applicable" answers and a low visual function score in Malawians is consistent with a similar findings in a Canadian population. ${ }^{8}$ We hypothesise that patients with low visual function adapt to their loss and simply no longer consider doing certain activities. Thus, the number of questions answered may be another measure of an individual's visual function.

This visual function test was easy to administer, at least as simple as administering distance visual acuity in the field. The visual function assessment could prove to be a valuable tool for field workers to identify and convince adults who would benefit significantly from surgery, but this still needs to be tested. It addition, it may help to identify those needing low vision care. ${ }^{12}$ Asking patients specific questions about what their vision allows them to do may increase their awareness of their own visual needs and the potential of surgery. Application of a visual function assessment by community based health workers (possibly traditional healers) may also assist these workers in recognising the need for intervention. It is our view that what a patient perceives as successful, (that is, what he can do after surgery compared with what he could do before) is of greater significance to him than his level of visual acuity. We hope that visual function questionnaires such as described in our study, with appropriate local adaptations, may prove useful in increasing cataract surgical uptake in a variety of locations in the future. 
This study was supported by the International Eye Foundation and the BC Centre for Epidemiologic and International Ophthalmology, for which we are grateful. We would like to thank Ms Newby Nkhata, Christoffel Blindenmission, $\mathrm{Mr}$ George Mekisini of the International Eye Foundation, and Mrs
Olga Mtambo, ophthalmic assistant at Queen Elizabeth Central Hospital, for their assistance with patient interviews and focus group discussions.

1 Bernth-Petersen, P. Visual functioning in cataract patients: methods of measuring and results. Acta Ophthalmol 1981;59:198-205.

2 Brenner MH, Curbow B, Javitt JC, et al. Vision change and quality of life in the elderly. Arch Ophthalmol 1993;111: $680-5$.

3 Steinberg EP, Tielsch JM, Schein OD, J et al. The VF-14, an index of functional impairment in patients with cataract. Arch Ophthalmol 1994;112:630-8. 4 Mangione CM, Phillips RS, Seddon JM, et al. Development functional status. Med Care 1992;30:1111-26.

5 Desai P, Reidy A, Minassian DC, et al. Gains from cataract surgery: visual function and quality of life. Br f Ophthalmol 1996;80:868-73.
6 Mangione CM, Phillips RS, Lawrence MG, et al. Improved visual function and attenuation of declines in health-related visual function and attenuation of declines in health-related
quality of life after cataract surgery. Arch Ophthalmol 1994; quality of life

7 Steinberg EP, Tielsch JM, Schein OD, J et al. National Study of Cataract Surgery Outcomes-variation in 4-month postoperative outcomes as reflected in multiple outcome measures. Ophthalmology 1994;101:1131-40.

8 Courtright P, Poon CI, Richards JSF, et al. Visual function among corneal disease patients waiting for penetrating keratoplasty in British Columbia. Ophthalmic Epidemiol 1998;5:13-20.

9 Ellwein LB, Fletcher A, Negrel AD, et al. Quality of life assessment in blindness prevention interventions. Int Ophthalmol 1995;18:263-8.

10 Fletcher AE, Ellwein LB, Selvaraj S, et al. Measurements of vision function and quality of life in patients with cataracts in southern India. Arch Ophthalmol 1997;115:767-74.

11 Scrimshaw SCM, Hurtado E. Rapid assessment procedures for nutrition and primary health care. United Nations University, Tokyo 1987.

12 World Health Organisation. Low vision care for the elderly: report of a workshop. Madrid, 4-6 July 1996, WHO/PBL/ 96.57. Geneva: WHO, 1996. 